\title{
AN EXCHANGE THEOREM FOR INDEPENDENCE STRUCTURES
}

\author{
C. J. H. MCDIARMID
}

ABSTRACT. I give a simple proof of the following result, which extends a result of $C$. Greene [1]. Let $X$ and $Y$ be sets in andependence structure $\mathcal{E}$ and let $X=X_{1} \cup X_{2}$ be a partition of $X$. Then there exists a partition $Y=Y_{1} \cup Y_{2}$ of $Y$ such that for $i=1,2, X_{i} \cap Y_{i}=\varnothing$ and $X_{i} \cup Y_{i}$ is in $\varepsilon$.

I give a simple proof of a result for independence structures (not necessarily finite) which extends a known result for matroids. The matroid result is stated and proved by C. Greene [1], and given a short proof by D. R. Woodall [3]. My proof is based on a simplification of Woodall's proof.

Theorem. Let $\mathcal{E}, \rho$ be an independence structure, let $X$ and $Y$ be sets in $E$, and let $X=X_{1} \cup X_{2}$ be a partition of $X$. Then there exists $a$ partition $Y=Y_{1} \cup Y_{2}$ of $Y$ such that for $i=1,2 X_{i} \cap Y_{i}=\varnothing$ and $X_{i} \cup Y_{i}$ is in $\tilde{G}$.

Note that if $\mathcal{E}$ has finite rank and $X$ and $Y$ are bases of $\mathcal{E}$, then it is immediate that $X_{1} \cup Y_{1}$ and $X_{2} \cup Y_{2}$ are bases of $E$, since

$$
\left|X_{1} \cup Y_{1}\right|+\left|X_{2} \cup Y_{2}\right|=\left|X_{1}\right|+\left|Y_{1}\right|+\left|X_{2}\right|+\left|Y_{2}\right|=|X|+|Y| \text {. }
$$

This is essentially the result proved in [1] and [3]. To prove the Theorem I use the following well-known lemma.

Lemma. Let $\mathcal{E}, \rho$ be an independence structure on a set $S$, let $X \epsilon$ $\xi$ and let $\mathcal{E}^{\prime}$ be the contraction of f from $X$, that is $\mathcal{G}^{\prime}=\{A \subseteq S \backslash X$ : $A \cup X \in \mathcal{E}\}$. Then $\mathcal{E}^{\prime}$ is an independence structure on $S \backslash X$, with rank function $\rho^{\prime}$, say, and for each finite subset $A$ of $S \backslash X$ there exists a finite subset $B$ of $S$ such that $\rho^{\prime} A=\rho(A \cup(X \cap B))-|X \cap B|$.

Received by the editors October 25, 1973 and, in revised form, November 1, 1973.

AMS (MOS) subject classifications (1970). Primary 05B 35. 
Proof of Lemma. Easy, using the finite character of $\varepsilon$.

Proof of Theorem. For $i=1,2$, let $\varepsilon_{i}, \rho_{i}$ be the contraction of $\varepsilon$ from $X_{i}$. We wish to show that $Y$ is in the sum $\varepsilon_{1} \vee \varepsilon_{2}$. Now this happens [2] if and only if for each finite subset $A$ of $Y, \rho_{1} A+\rho_{2} A \geq|A|$. But for each finite subset $A$ of $Y$, and some finite set $B$, using the submodularity of $\rho$,

$$
\begin{aligned}
\rho_{1} A+\rho_{2} A & =\rho\left(A \cup\left(X_{1} \cap B\right)\right)-\left|X_{1} \cap B\right|+\rho\left(A \cup\left(X_{2} \cap B\right)\right)-\left|X_{2} \cap B\right| \\
& \geq \rho(X \cap B)+\rho A-|X \cap B|=|A| .
\end{aligned}
$$

\section{REFERENCES}

1. C. Greene, A multiple exchange property for bases, Proc. Amer. Math. Soc. 39 (1973), 45-50. MR $47 \# 56$.

2. J. S. Pym and H. Perfect, Submodular functions and independence structures, J. Math. Anal. Appl. 30 (1970), 1-31. MR 41 \#8269.

3. D. R. Woodall, An exchange theorem for bases of matroids, J. Combinatorial Theory 16 (1974), 227-228.

CORPUS CHRISTI COLLEGE, OXFORD, ENGLAND 